\title{
Efficacy of Lopinavir Combined with Doxycycline for Treatment of COVID-19: A Retrospective Comparison of Two Cohorts
}

\author{
FERHAT ARSLAN ${ }^{1}$, Ali Mert ${ }^{2}$, Mehmet Bayram², Sacit Icten ${ }^{3}$, Pinar Ergen $^{3}$, Ozlem \\ Aydin $^{3}$, Ayse Ucişik ${ }^{3}$, Fatma Yilmaz Karadag ${ }^{3}$, Hülya Caskurlu ${ }^{1}$, Bahadir Ceylan ${ }^{2}$, Selda \\ Aydin², Muhammed Akkoyunlu², Mustafa Duger², Okan Derin², Abdullah Kansu², Sedef \\ Basgonul $^{2}$, Tulin Akarsu ${ }^{3}$, Hasan Kocoglu ${ }^{1}$, Yasemin $\mathrm{Cag}^{1}$, and Haluk Vahaboglu ${ }^{1}$ \\ ${ }^{1}$ Medeniyet Universitesi Goztepe Egitim ve Arastirma Hastanesi \\ ${ }^{2}$ Istanbul Medipol University \\ ${ }^{3}$ Goztepe Training and Research Hospital
}

July 16, 2020

\begin{abstract}
Introduction: Though there is currently no approved treatment for COVID-19, potential medications include lopinavir and doxycycline. Lopinavir is a broad-spectrum protease inhibitor successfully used during the SARS outbreak. Doxycycline is an antibiotic with immunomodulatory effects, which successfully treats dengue hemorrhagic fever. Comparing lopinavir combined with doxycycline with other commonly implemented COVID-19 treatment regimens is of medical significance. Methods: We conducted a retrospective study comparing two cohorts. One cohort strictly adhered to a treatment protocol of lopinavir combined with doxycycline (Lop/Dox), while the other cohort (Others) applied protocols based on combinations of favipiravir, hydroxychloroquine, and azithromycin. The outcomes evaluated were death from coronavirus and intubation. Results: During the study period, 161 and 114 patients were followed in the Lop/Dox cohort and the Others cohort, respectively. The Lop/Dox cohort was older and had lower $\mathrm{O} 2$ saturation at admission. The proportion of patients needing intubation did not differ between the cohorts (Lop/Dox, 15[?]5\%; Others, 16[?]7\%). The overall fatality rate likewise did not differ (12[?]4\%) in Lop/Dox and $8[?] 7 \%$ in the Others cohort). Older age and elapsed time between the onset of symptoms and hospitalization, O2 saturation at admission, and the lymphocyte to white blood cell ratio were independent risk factors for poor outcomes of the illness. Conclusion: This study showed that lopinavir plus doxycycline therapy is an effective treatment amongst moderate to severe COVID-19 patients.
\end{abstract}

Efficacy of Lopinavir Combined with Doxycycline for Treatment of COVID-19: A Retrospective Comparison of Two Cohorts

\section{Running Title : Lopinavir versus other antivirals in COVID-19 patients}

Ferhat Arslan, ${ }^{1}$ Ali Mert, ${ }^{2}$ Mehmet Bayram, ${ }^{3}$ Sacit Icten, ${ }^{3}$ Pinar Ergen ${ }^{4}$, Ozlem Aydin ${ }^{4}$, Ayse Canan Ucisik $^{4}$, Fatma Yilmaz-Karadag ${ }^{4}$, Hulya Caskurlu ${ }^{1}$, Bahadır Ceylan ${ }^{2}$, Selda Aydın ${ }^{2}$, Muhammed Emin Akkoyunlu $^{3}$, Mustafa Düger ${ }^{3}$, Okan Derin ${ }^{2}$, Abdullah Kansu ${ }^{3}$, Sedef Başgönül ${ }^{2}$, Tulin Akarsu-Ayazoglu ${ }^{5}$, Hasan Kocoglu ${ }^{5}$, Yasemin $\mathrm{Cag}^{1}$, Haluk Vahaboglu ${ }^{1}$,

${ }^{1}$ Department of Infectious Diseases and Clinical Microbiology, Istanbul Medeniyet University Faculty of Medicine, Istanbul, Turkey

${ }^{2}$ Department of Infectious Diseases and Clinical Microbiology, Istanbul Medipol University Faculty of Medicine, Istanbul, Turkey 
${ }^{2}$ Department of Pulmonary Medicine, Istanbul Medipol University Faculty of Medicine, Istanbul, Turkey

${ }^{3}$ Department of Pulmonary Medicine, Istanbul Medeniyet University Faculty of Medicine, Istanbul, Turkey

${ }^{4}$ Department of Infectious Diseases and Clinical Microbiology, İstanbul Medeniyet University, Göztepe Training and Research Hospital, İstanbul, Turkey.

${ }^{5}$ Department of Anesthesiology and Reanimation, Istanbul Medeniyet University Faculty of Medicine, Istanbul, Turkey

\section{Corresponding Author}

Assoc. Prof. Ferhat Arslan, M.D.

Istanbul Medeniyet University

Infectious Diseases and Clinical Microbiology

Eğitim Mah. Dr. Erkin Cad. Kadıköy/İstanbul 34722

Phone : +90 (216) 5664000

Fax : +90216566 6614

E-mail:ferhatarslandr@hotmail.com

Word count: 1996

\section{What is already known about this subject}

- Currently, there is no approved treatment for COVID-19

- The main proteases of SARS-COV and SARS-COV-2 are $96 \%$ identical, and in silico

- studies have shown that HIV protease inhibitors can inhibit coronavirus protease

- Lopinavir-ritonavir may still be a potential therapeutic agent against Covid-19.

\section{What this study adds}

- Doxycycline is a strong inducer of suppressors of cytokine signaling proteins (SOCS)

- and is successfully used among dengue hemorrhagic fever patients

- Lopinavir in combination doxycycline therapy seems effective, especially soon after

- hospital admission for COVID-19.

\section{ABSTRACT}

\section{Introduction:}

Though there is currently no approved treatment for COVID-19, potential medications include lopinavir and doxycycline. Lopinavir is a broad-spectrum protease inhibitor successfully used during the SARS outbreak. Doxycycline is an antibiotic with immunomodulatory effects, which successfully treats dengue hemorrhagic fever. Comparing lopinavir combined with doxycycline with other commonly implemented COVID-19 treatment regimens is of medical significance.

\section{Methods:}

We conducted a retrospective study comparing two cohorts. One cohort strictly adhered to a treatment protocol of lopinavir combined with doxycycline (Lop/Dox), while the other cohort (Others) applied protocols based on combinations of favipiravir, hydroxychloroquine, and azithromycin. The outcomes evaluated were death from coronavirus and intubation.

\section{Results:}

During the study period, 161 and 114 patients were followed in the Lop/Dox cohort and the Others cohort, respectively. The Lop/Dox cohort was older and had lower $\mathrm{O}_{2}$ saturation at admission. The proportion of 
patients needing intubation did not differ between the cohorts (Lop/Dox, 15[?]5\%; Others, 16[?]7\%). The overall fatality rate likewise did not differ (12[?]4\%) in Lop/Dox and $8[?] 7 \%$ in the Others cohort). Older age and elapsed time between the onset of symptoms and hospitalization, $\mathrm{O}_{2}$ saturation at admission, and the lymphocyte to white blood cell ratio were independent risk factors for poor outcomes of the illness.

\section{Conclusion:}

This study showed that lopinavir plus doxycycline therapy is an effective treatment amongst moderate to severe COVID-19 patients.

Key words : Lopinavir; Faviprevir ; Doxycycline ; COVID-19 ; Coronavirus ; Hydroxychloroquine

\section{INTRODUCTION}

The coronavirus disease (COVID-19) pandemic, caused by the novel severe acute respiratory syndrome coronavirus 2 (SARS-CoV-2), has collapsed the infrastructure and health care systems of countries worldwide. ${ }^{1}$ Currently, there is no approved treatment for COVID-19. Hence, efforts for the development and repurposing of efficient and effective medications are ongoing. ${ }^{2}$

A protease inhibitor, lopinavir (LPV), had been associated with reduced mortality and morbidity rate in clinical studies during the SARS and MERS outbreaks. ${ }^{3,4}$ The main proteases of SARS-COV and SARSCOV-2 are $96 \%$ identical, and in silico studies have shown that HIV protease inhibitors can inhibit coronavirus protease ${ }^{5}$ Clinical trials of the antiviral efficacy of drugs, including LPV, have been registered and are ongoing with regard to COVID-19. ${ }^{6}$ However, while randomized clinical trials are continuing, observational data are also urgently needed to assess the efficacy and safety of this antiviral drug in COVID-19 treatment.

The scientific committee of the Turkish Health Ministry currently recommends hydroxychloroquine (HCQ) plus azithromycin or favipiravir (FVP) in the treatment of COVID-19 patients. The recommendations of the committee are widely applied. However, to our knowledge at least one Turkish medical center exclusively uses LPV in combination with the medication doxycycline in the management of moderate to severe COVID-19 cases. Comparing clinical outcomes of various anti-viral regimens is of scientific interest.

In this study, we compared the outcomes between patients who were treated with LPV in combination with doxycycline at one medical center and the patients treated with other antivirals regimens at another medical center.

\section{OBJECTIVES}

\section{Study design}

We conducted a retrospective study comparing two cohorts. One cohort strictly adhered to a treatment protocol (LPV plus doxycycline) while the other cohort applied protocols recommended by the scientific committee of the Turkish Heath Ministry. This study included patients diagnosed with COVID-19 induced pneumonia from March 21 to Apr 23, 2020, at two university hospitals located on distinct sides of the Istanbul province in Turkey.

The ethics committee of the Istanbul Medipol University and the Health Ministry COVID-19 Research Committee of Turkey approved this study (No: 10840098-604.01.01-E.14713). Ethics committee waived obtaining informed consent.

\section{Centers, Patients and Definitions}

We hereafter will describe centers according to the treatment protocol instead of the center name. Specifically, the center administering lopinavir plus doxycycline is referred to as "Lop/Dox," and that administering a range of treatment protocols approved by the Health Ministry is referred to as "Others." 
Cases were patients $>18$ years old who were hospitalized and found positive for the novel SARS-CoV-2 RNA following nasopharyngeal swab testing using RT-PCR. The Lop/Dox cohort consisted of moderate to severe patients, since mild patients were home isolated with a different treatment protocol. The Others cohort included more mild cases, as well as moderate to severe cases.

Study covariates included age (years), gender, lymphocyte count, white blood cell count, body temperature $\left({ }^{\circ} \mathrm{C}\right)$ at admission, $\mathrm{O}_{2}$ saturation $(\%)$ at admission, respiration rate per minute at admission, the elapsed time between the onset of symptoms and hospitalization, history of hypertension, and ACE inhibitors usage. The study outcome measures are described below. We extracted all data from medical records.

FPV $600 \mathrm{mg}$, HCQ $200 \mathrm{mg}$, LPV $200 \mathrm{mg}$, and doxycycline $100 \mathrm{mg}$, all were administered twice daily via the PO route. Azithromycin was administered $250 \mathrm{mg}$ PO once daily. For FVP, HCQ and azithromycin loading doses were given on the first day.

We analyzed the need for intubation as the primary outcome and in-hospital mortality as a secondary outcome. The need for intubation seems to be a more sensitive measure to compare the efficacy of the two antiviral drugs in this setting. This is because, following the intubation, patients are transferred to the ICU; hence, new modalities and risks appear.

\section{Statistical Analysis}

We used the open-source platform $\mathrm{R}$ for statistical analyses (R Foundation for Statistical Computing, Vienna, Austria), including appropriate packages such as rms, Boruta, and compare groups. ${ }^{7-9}$ We present descriptive statistics as medians and interquartile ranges for continuous variables that violate normality, and present normally distributed continuous variables as means. We present categorical variables as frequencies and percentages. All univariate statistics were two-sided.

We selected appropriate tests for univariate comparisons depending on the nature of the variable. Accordingly, we used Student's t-test or the Kruskall-Wallis test for continuous variables and chi-squared or Fisher exact tests for categorical variables.

Time to event analysis and cumulative incidences were plotted by the survminer package. The failure event was intubation, and the date variable was the time elapsed between hospitalization and intubation. Statistical significance was estimated by the log-rank test.

We constructed a full model by including variables important in univariate comparisons (at a significance level of $\mathrm{p}<0.05$ ), as well as variables important based on medical knowledge. We then selected relevant variables using the Boruta package. We used a random forest algorithm with default attributes in the elimination procedure and used a multiple imputation algorithm to impute missing observations. More specifically, we imputed missing observations five times and then combined effect estimates via the Hmisc package. We also tested medically important variables one by one in the final model and decided on their inclusion according to the calibration plot and performance indices. We internally validated the final model using bootstrap resampling evaluated according to Somers' Dxy rank correlation and the C-index (the concordance probability).

Finally, we also constructed a nomogram using the linear predictions of the final model for the intubation outcome.

\section{RESULTS}

During the study period, 161 and 114 patients were followed in the Lop/Dox cohort and the Others cohort, respectively. Table 1 displays the baseline characteristics of the cohorts.

Briefly, most severity factors were more pronounced in the Lop/Dox cohort. For example, age was significantly higher (55[?]0 [46[?]0, 64[?]8] vs 61[?]0 [48[?]0, 72[?]0], years) in the Lop/Dox cohort, which is an important factor associated with adverse outcomes. Respiration rate per minute and ACE II inhibitor usages were 
likewise all high in the Lop/Dox cohort, while $\mathrm{O}_{2}$ saturation was lower. However, the number of patients that needed intubation did not differ between the cohorts (Lop/Dox, 25 (15[?]5\%); Others, 19 (16[?]7\%)).

The overall fatality rates in the Lop/Dox and Others cohorts were $12[?] 4 \%(20 / 161)$ and $8[?] 7 \%(10 / 114)$, respectively. The fatality rates among intubated patients in the Lop/Dox and Others cohorts were $68 \%$ $(17 / 25)$ and $52.6 \%(10 / 19)$, respectively $(p$-value $=0.79)$. Neither fatality rate comparison was statistically significant.

A univariate comparison of baseline risk factors between patients who needed intubation to maintain $\mathrm{O}_{2}$ saturation and those not requiring intubation is displayed in Table 2.

Among the evaluated factors, age, white blood cell (WBC) count, lymphocyte to $\mathrm{WBC}$ ratio, $\mathrm{O}_{2}$ saturation, respiration rate per minute, the elapsed time between the onset of symptoms to hospitalization, and hypertension were statistically significant. Figure 1 displays the cumulative incidence of intubated patients in the Lop/Dox and Others cohort.

The numbers of intubated patients did not differ between the two cohorts, although severity parameters were more unfavorable in the Lop/Dox cohort.

Estimated effects from multivariate models are displayed in Table 3.

Briefly, three variables were retained in the final model when evaluating fatality as an outcome, while four variables were retained for the intubation outcomes. Specifically, age, oxygen saturation at hospital admission, and elapsed time between the onset of symptoms and hospitalization were the covariates associated with COVID-19 fatality. Age was not associated with intubation, in contrast to the lymphocyte to WBC count ratio, which was associated with intubation. We also constructed a nomogram that displays the relative importance of predictive covariates and the estimated risk of intubation, as shown in Figure 2.

\section{DISCUSSION}

We conducted a comparative study between two medical centers, which demonstrated that lopinavir in combination with doxycycline is as effective as the FVP, HCQ, and azithromycin combination regimen. Lopinavir is a broad-spectrum protease inhibitor that was successfully implemented during the SARS and MERS outbreaks. ${ }^{10}$ In silico docking studies have also indicated that lopinavir can inhibit SARS-CoV-2 protease. ${ }^{11}$ Therefore, lopinavir is a widely recognized experimental alternatives to more established COVID19 medications. Recently, a randomized controlled study compared lopinavir to standard-care therapy among COVID-19 patients with a median of 13 days of delay from the onset of symptoms. ${ }^{12}$ Although the lopinavir arm displayed an apparent benefit at 14 days, the difference did not maintain statistical significance by 28 days. However, this study included patients at the late stage of the disease. Pulmonary damage resulting from excessive inflammation independent of viral activity in the later stages of the disease reveals the importance of effective early intervention using antivirals. ${ }^{13,14}$

Postmortem studies indicate that pulmonary damage might be related to dysregulated inflammation (rather than viral activity) in the alveoli caused by accumulated highly cytotoxic lymphocytes and inflammatory cells. ${ }^{15}$ Therefore, supporting the regulation of cytokine production is thought to be important in COVID-19 treatment. Doxycycline is a strong inducer of suppressors of cytokine signaling proteins (SOCS) ${ }^{16}$ and is successfully used among dengue hemorrhagic fever patients. ${ }^{17}$ We supplemented lopinavir with doxycycline for its immunomodulatory effect as well as its antibacterial activity, especially against pulmonary pathogens like mycoplasma. We note that, before test results become available, making a differential diagnosis between COVID-19 pneumonia and similarly presenting illnesses might be challenging in some cases, such as Mycoplasma pneumonia.

The patients included in our study were hospitalized after a median of five days from the onset of initial signs and symptoms; this was true in both medical centers comprising our study. Interestingly, the elapsed time between the onset of symptoms and hospitalization was inversely correlated with adverse outcomes. 
This inverse correlation probably reflects the speed of the disease trajectory from the onset of symptoms to deterioration. This finding is surprising because the intuitive perception is that a patient admitted to the hospital and receiving medications early would be more likely to have an advantageous outcome. However, in real life, most patients with mild symptoms do not seek an immediate medical investigation, and this may skew the observed results.

We found that advanced age was one of the most critical risk factors for fatality regardless of the treatment regimen. This finding is consistent with previously published case series from China and the United States. ${ }^{18}$ Sex (i.e. male gender) previously considered an important predictive factor for COVID-19 mortality, was not associated with mortality based on the univariate and multivariate analyses in our cohort. In the literature, elevated inflammation biomarkers such as C-reactive protein, ferritin, and increased neutrophilto-lymphocyte ratio have been associated with death from the COVID-19 disease. ${ }^{19}$ Secondary bacterial infections are inevitable in COVID-19 patients with severely damaged bronchial mucosa, especially after intubation; this is true among patients with a normal respiratory system or among patients with nosocomial flora pathogens. For instance, in three of our patients, Acinetobacter baumanniirelated ventilator-associated pneumonia was developed in an ICU that treated COVID-19 with antibiotics. In such a case, nonspecific inflammation biomarkers generally increase.

In the face of this new and challenging disease, our goal is to develop a risk assessment tool that strengthens the clinician's resources in treating patients. However, the lack of optimal selection of multiple parameters make risk assessment tools difficult to use. We therefore recommend our nomogram, which consists of symptom duration, vital signs, and blood parameters to ensure rapid triage in the management of patients at risk.

Our study has limitations, including its retrospective nature and some violations of comparability at the two hospital settings. The compliance of outpatient treatments could not be followed up in patients discharged early. Unrecorded data about the patients who remained hospitalized, especially at the ICU, after the study was complete may cause the results to be biased.

In conclusion, although there are different treatment protocols between centers, we identified classic risk factors, such as age and oxygen saturation, as the determinants of COVID-19 related pneumonia prognosis. Hence, until a drug or drug combination is available that is rigorously evidence-based, lopinavir in combination doxycycline therapy seems effective, especially soon after hospital admission for COVID-19.

\subsubsection{Acknowledgments}

During the epidemic of COVID-19, we would like to thank all our healthcare professionals, governors for their supports, and the Turkish people who comply with our warnings during the national lockdown.

\subsubsection{Declarations of interest : None}

\subsubsection{References}

1. Wu Z, McGoogan JM. Characteristics of and Important Lessons From the Coronavirus Disease 2019 (COVID-19) Outbreak in China: Summary of a Report of 72314 Cases From the Chinese Center for Disease Control and Prevention. JAMA . Published online February 24, 2020. doi:10.1001/jama.2020.2648

2. Scavone C, Brusco S, Bertini M, et al. Current pharmacological treatments for COVID-19: what's next? Br J Pharmacol . Published online April 24, 2020. doi:10.1111/bph.15072

3. Chan KS, Lai ST, Chu CM, et al. Treatment of severe acute respiratory syndrome with lopinavir/ritonavir: a multicentre retrospective matched cohort study. Hong Kong Med J Xianggang Yi Xue Za Zhi . 
2003;9(6):399-406.

4. Chu C, Cheng V, Hung I, et al. Role of lopinavir/ritonavir in the treatment of SARS: initial virological and clinical findings. Thorax . 2004;59(3):252-256. doi:10.1136/thorax.2003.012658

5. Ortega JT, Serrano ML, Pujol FH, Rangel HR. Unrevealing sequence and structural features of novel coronavirus using in silico approaches: The main protease as molecular target. EXCLI J . 2020;19:400-409. doi:10.17179/excli2020-1189

6. Stebbing J, Phelan A, Griffin I, et al. COVID-19: combining antiviral and anti-inflammatory treatments. Lancet Infect Dis . 2020;20(4):400-402. doi:10.1016/S1473-3099(20)30132-8

7. Harrell Jr FE. Rms: Regression Modeling Strategies .; 2019. https://cran.r-project.org/package=rms

8. Kursa M, Softw WR-JS, undefined 2010. Feature selection with the Boruta package. academia.edu . http://www.academia.edu/download/32056178/v36i11.pdf

9. Subirana I, Sanz H, Vila J. Building Bivariate Tables: The \{compareGroups $\}$ Package for $\{$ R $\}$. J Stat Softw . 2014;57(12):1-16.

10. Yao T-T, Qian J-D, Zhu W-Y, Wang Y, Wang G-Q. A Systematic Review of Lopinavir Therapy for SARS Coronavirus and MERS Coronavirus-A Possible Reference for Coronavirus Disease-19 Treatment Option. $J$ Med Virol . 2020;(February):1-8. doi:10.1002/jmv.25729

11. Lin S, Shen R, He J, Li X, Guo X. Molecular modeling evaluation of the binding effect of ritonavir, lopinavir and darunavir to severe acute respiratory syndrome coronavirus 2 proteases. bioRxiv . Published online 2020:2020.01.31.929695. doi:10.1101/2020.01.31.929695

12. Cao B, Wang Y, Wen D, et al. A Trial of Lopinavir-Ritonavir in Adults Hospitalized with Severe Covid-19. N Engl J Med . Published online March 2020:NEJMoa2001282. doi:10.1056/NEJMoa2001282

13. Hogan RJ, Zhong W, Usherwood EJ, Cookenham T, Roberts AD, Woodland DL. Protection from respiratory virus infections can be mediated by antigen-specific CD4(+) T cells that persist in the lungs. $J$ Exp Med . 2001;193(8):981-986. doi:10.1084/jem.193.8.981

14. Cao W, Li T. COVID-19: towards understanding of pathogenesis. Cell Res . 2020;30(5):367-369. doi:10.1038/s41422-020-0327-4

15. Xu Z, Shi L, Wang Y, et al. Pathological findings of COVID-19 associated with acute respiratory distress syndrome. Lancet Respir Med . 2020;8(4):420-422. doi:10.1016/S2213-2600(20)30076-X

16. Karlsen AE, Ronn SG, Lindberg K, et al. Suppressor of cytokine signaling 3 (SOCS-3) protects $\beta$-cells against interleukin-1 $\beta$ - and interferon- $\gamma$-mediated toxicity. Proc Natl Acad Sci U S A . 2001;98(21):1219112196. doi:10.1073/pnas.211445998

17. Fredeking T, Zavala-Castro J, Gonzalez-Martinez P, et al. Dengue Patients Treated with Doxycycline Showed Lower Mortality Associated to a Reduction in IL-6 and TNF Levels. Recent Patents Anti-Infect Drug Disc . 2015;10(1):51-58. doi:10.2174/1574891x10666150410153839

18. Richardson S, Hirsch JS, Narasimhan M, et al. Presenting Characteristics, Comorbidities, and Outcomes Among 5700 Patients Hospitalized With COVID-19 in the New York City Area. JAMA. Published online April 22, 2020. doi:10.1001/jama.2020.6775

19. Zhou F, Yu T, Du R, et al. Clinical course and risk factors for mortality of adult inpatients with COVID-19 in Wuhan, China: a retrospective cohort study. Lancet Lond Engl . 2020;395(10229):1054-1062. doi:10.1016/S0140-6736(20)30566-3 


\section{Figure legends:}

Figure 1. Cumulative incidence plot of Lop/Dox and Others cohorts. Events are shown over the $\mathrm{x}$ axis of the plot.

Figure 2. A nomogram for outcome intubated. Total points obtained by the sum of the individual points will predict the risk of intubation.
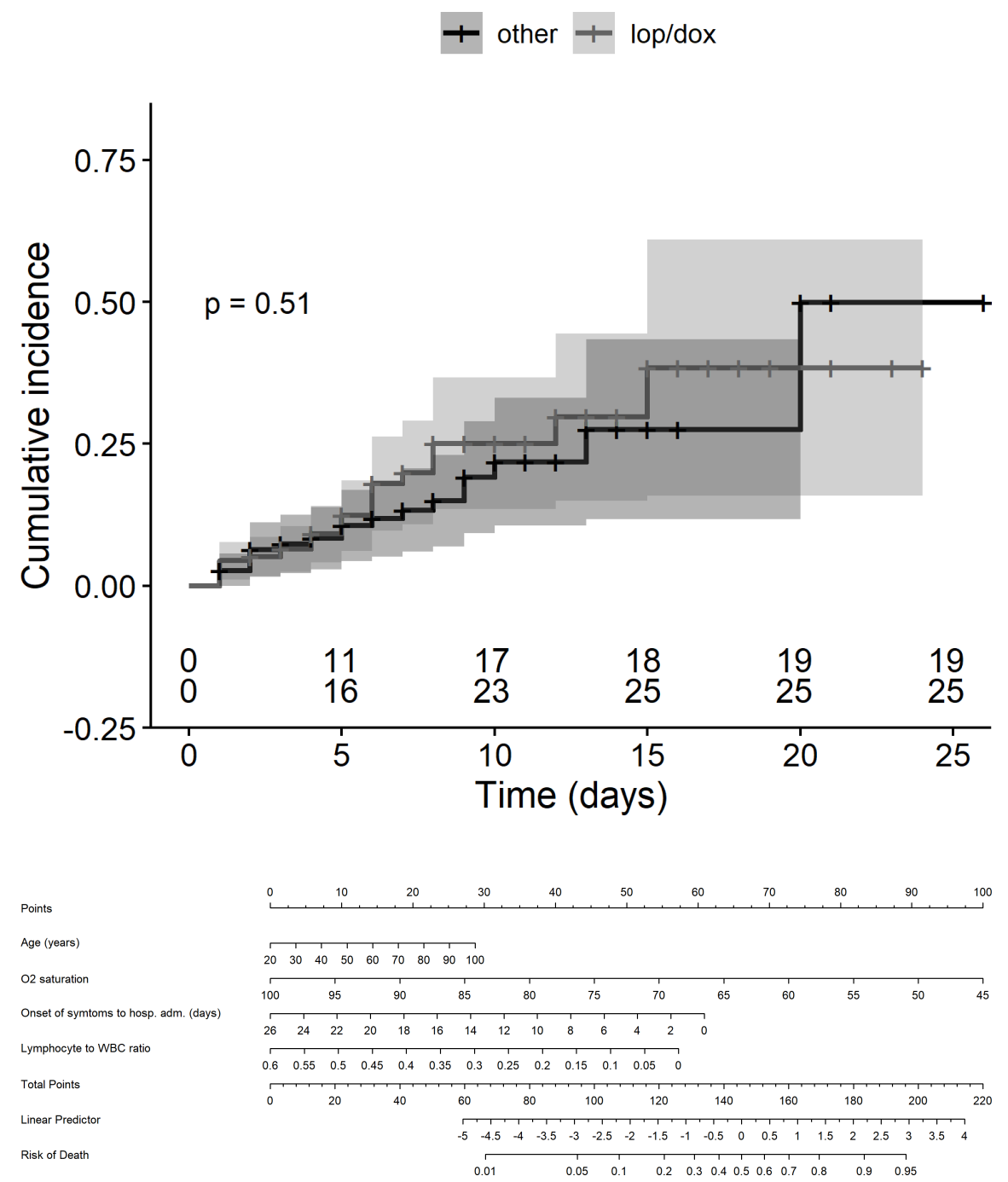

\section{Hosted file}

Table_1.docx available at https://authorea.com/users/342961/articles/469717-efficacyof-lopinavir-combined-with-doxycycline-for-treatment-of-covid-19-a-retrospectivecomparison-of-two-cohorts

\section{Hosted file}

Table_2.docx available at https://authorea.com/users/342961/articles/469717-efficacyof-lopinavir-combined-with-doxycycline-for-treatment-of-covid-19-a-retrospectivecomparison-of-two-cohorts 


\section{Hosted file}

Table_3.docx available at https://authorea.com/users/342961/articles/469717-efficacyof-lopinavir-combined-with-doxycycline-for-treatment-of-covid-19-a-retrospectivecomparison-of-two-cohorts 